\title{
Blacks Have a Greater Risk of Stillbirth Than Whites Following a Cesarean, and Higher Stillbirth Recurrence
}

\begin{abstract}
Women whose previous delivery had been by cesarean had a rate of stillbirth in their subsequent pregnancy similar to that of women who had had a vaginal delivery, according to a longitudinal study of Missouri births. ${ }^{1}$ However, black women had a higher rate of stillbirth than white women, and blacks who had had a cesarean were more likely to have a stillbirth than were blacks who had delivered vaginally. A second study by the same team of researchers using the same data found that the rate of stillbirth in the second pregnancy was higher for those who had had a stillbirth than for those who had not. ${ }^{2}$ Blacks experienced a higher rate of recurrence of stillbirth than whites.
\end{abstract}

\section{Stillbirth Following Cesarean Delivery}

The rate of cesarean deliveries in the United States has been increasing since 1996, and one in three births among black women are by cesarean, which is the highest rate for any racial group. Blacks also have a greater risk of having stillbirths, and the rising use of cesarean delivery may be disproportionately affecting this group.

The Missouri cohort data set for the period 1978-1997 identifies and links newborns to their biological mothers. To assess the association between cesarean delivery and stillbirth in subsequent pregnancy, the researchers examined the records of women who had had two consecutive singleton births at 20 or more weeks of gestation; infants with congenital abnormalities were excluded. A stillbirth was defined as intrauterine fetal death at a gestational age of 20 or more weeks.

Because this study focused on the outcome of the second pregnancy, data on maternal characteristics at the later pregnancy were used; these included age, educational level, race, marital status, cigarette smoking, adequacy of prenatal care (using an index based on the trimester in which care began, number of visits and gestational age at birth) and body mass index. The occurrence of obstetric complications in the second pregnancy was also examined. Chi-square and t tests analyzed differences in these characteristics between subgroups.
Multivariable logistic regression analysis was used to assess associations between cesarean delivery and subsequent stillbirth; regression models controlled for the foregoing maternal characteristics, as well as for parity, interpregnancy interval and year of birth.

Of the 396,441 women in the sample, $82 \%$ delivered vaginally and $18 \%$ by cesarean in their first pregnancy. Compared with the proportion among women who had had a vaginal delivery, higher proportions of those with a previous cesarean had 12 or more years of education ( $86 \%$ vs. $81 \%$ ) and were 35 or older ( $10 \%$ vs. $7 \%$ ), white ( $88 \%$ vs. $84 \%$ ), married ( $84 \%$ vs. $80 \%$ ) and overweight or obese $(31 \%$ vs. $21 \%$ ). A higher proportion of these women had received adequate prenatal care $(51 \%$ vs. $40 \%$ ), while a lower proportion had smoked during the second pregnancy ( $23 \%$ vs. $26 \%$ ). Greater proportions of women who had had a cesarean experienced medical complications in their second pregnancies, including anemia, cardiac disease, diabetes, chronic hypertension, preeclampsia, eclampsia and placental disorders. Overall, $18 \%$ of women who had undergone this procedure had any medical complication, whereas 14\% of those who had not had it suffered a complication. The cesarean group also had a slightly higher rate of preterm births, but there was no difference between the groups in the proportion of infants who were small for gestational age.

In all, 1,612 pregnancies ended in stillbirths; no difference was found in the stillbirth rate between women who had had vaginal and those who had had cesarean delivery (4.4 per 1,000 for each group). However, blacks had a significantly higher rate of stillbirth than whites (7.2 vs. 3.6 per 1,000). Furthermore, while the rate of stillbirth did not differ between whites who had had a vaginal delivery and those who had undergone a cesarean, blacks who had had a cesarean delivery experienced a higher rate of stillbirth than those who had had a vaginal delivery (9.3 vs. 6.8 per 1,000). The adjusted analyses confirmed that black women with a previous cesarean had a higher risk of stillbirth than blacks who had delivered vaginally (odds ratio, 1.4). Further analysis by gestational age found a higher risk among blacks only for 35 or more weeks (1.4); the same risk for stillbirth was found when both gestational age and occurrence of medical complications were controlled for.

A major strength of the study, the researchers note, is the robust data set, which is considered a national standard. Limitations include scarce data on placental abnormalities and causes of stillbirth, and the aggregation of different infant cohorts. The researchers believe that the upsurge in cesarean deliveries over the last decade may be caused by changes in physician behavior and institutional practices, and that these changes may be a factor in black women's increased rates of both cesareans and stillbirth. They suggest that future research should examine whether there is a causal relationship between undergoing a cesarean and having a subsequent stillbirth.

\section{Race and Stillbirth Recurrence}

In the second study, the researchers examined whether race was a determinant of stillbirth recurrence. They looked at women who had had consecutive singleton births in which the fetus was between 20 and 44 weeks of gestational age. Analyses were similar to those conducted in the other study.

The sample included 404,180 women, of whom $99.5 \%$ had had a live birth and $0.5 \%$ had had a stillbirth in the first pregnancy. The two groups differed significantly in all characteristics but maternal age and educational level. Compared with the proportions among women who had not had a previous stillbirth, higher proportions of those who had had one were black (20\% vs. 14\%), unmarried (22\% vs. $19 \%$ ) and overweight or obese (28\% vs. 23\%). Higher proportions had smoked during pregnancy (28\% vs. 26\%) and had received adequate prenatal care (58\% vs. $42 \%$ ). These women also had higher rates of nearly all medical complications in their second pregnancies (anemia being the exception); 30\% experienced at least one complication, compared with $20 \%$ of women who had not had a stillbirth. 
Among the women in the sample, 1,929 had a stillbirth in the second pregnancy. The rate of stillbirth was significantly different between women who had had an earlier stillbirth and those who had not (22.7 vs. 4.7 per 1,000$)$. Rates were dramatically different depending on race: Black women who had experienced earlier stillbirth and those who had not had rates of 35.9 and 7.6 per 1,000 , respectively, while white women had rates of 19.1 and 4.2 per 1,000 , respectively. The overall likelihood of stillbirth was significantly elevated for women with a previous stillbirth (odds ratio, 4.7). Among these women, the risk of stillbirth recurrence was higher for blacks than for whites (2.6) after potential confounders were controlled for.

According to the researchers, strengths of this study are that it is population-based and that, unlike previous studies, it accounts for the effect of maternal body mass index, which is a critical cause of adverse pregnancy outcomes. However, the research also has a number of limitations: It did not assess the influ-

ence of maternal and fetal medical conditions, and there were few cases of stillbirths available for the racial analysis.

The researchers assert that there is a critical need to explore the risk profiles of women and to develop public health programs to ameliorate the racial disparity in the rate of stillbirth recurrence. They suggest that these programs include counseling and possibly changes in clinical practice to better serve the differing needs of patients. While this study highlights the need for a more comprehensive research agenda in this area, the researchers believe that their findings "will contribute to the enhancement of our current understanding of stillbirth recurrence, an area that has been poorly researched."-J. Thomas

\section{REFERENCES}

1. Salihu HM et al., Risk of stillbirth following a cesarean delivery: black-white disparity, Obstetrics $\&$ Gynecology, 2006, 107(2, pt. 1):383-390.

2. Sharma PP et al., Is race a determinant of stillbirth recurrence? Obstetrics \& Gynecology, 2006, 107(2, pt. 1):391-397.

\section{Under Texas Parental Notification Law, Minors' Abortion Rate Falls, but Risk of Late Abortion Rises}

Abortion rates fell among all teenagers in Texas after a parental notification law for minors took effect in the state at the beginning of 2000 , but declines among young women who were subject to the law were greater than declines among those who were not. ${ }^{1}$ However, among young women who had an abortion, those who had become pregnant 3-6 months before their 18th birthday were more likely to terminate their pregnancy during the second trimester than were those who had conceived after their 18th birthday.

Early studies of the effect of parental involvement laws on minors' pregnancy outcomes yielded conflicting findings, possibly because substantial numbers of young women were able to obtain abortions in neighboring states that had no law. However, the number of states enforcing parental involvement laws has doubled since the early 1990s; minors now have to travel much greater distances to obtain an abortion. Given this situation, such laws may lead to higher rates of birth and delayed abortion among women younger than 18 .

To examine this issue, researchers used data from birth and abortion certificates obtained from the Texas Department of State Health Ser- vices to compare changes in rates of abortion and birth among minors before and after the law took effect. Because these rates have been declining nationally and in Texas since the early 1990s, the researchers used rate changes among 18-year-olds as proxies for the changes that would have been expected without the law.

In 1998-1999, minors in Texas had an average of 5,769 conceptions each year that ended in abortion and 28,096 that ended in birth. In 2000-2002, those figures were 4,661 and 13,174 , respectively.

Rates of abortion among 15-17-year-olds ranged from 6.5 to 18.7 per 1,000 women in the earlier period, and from 5.4 to 14.5 during the later period. The rate for 18 -year-olds was 27.7 per 1,000 in 1998-1999 and 15.8 per 1,000 in 2000-2002. Between the two periods, the abortion rate fell by $22 \%$ among 17 year-olds, by $25 \%$ among 16 -year-olds and by $18 \%$ among 15 -year-olds; the rate among 18 year-olds decreased by $7 \%$. In comparison with the decline among 18-year-olds, those among younger women were $11-20 \%$ greater.

Birthrates among 15-17-year-olds ranged from 33.6 to 88.2 per 1,000 women in 1998-1999 and from 30.5 to 83.2 per 1,000 in 2000-2002. The rate for 18-year-olds was 117.9 per 1,000 in 1998-1999 and 112.4 per 1,000 in 2000-2002. Between the two periods, the birthrate decreased by $6 \%$ among 17 year-olds, by $9 \%$ among 16-year-olds and by 9\% among 15-year-olds; the rate among 18year-olds declined by $5 \%$. In comparison with the decline among 18-year-olds, those among 15-year-olds and 16-year-olds were significantly greater (by 5\% in each case).

To compensate for large differences in rates of abortion and birth between older and younger teenagers, the researchers conducted additional analyses limited to teenagers who became pregnant within a few months before or after their 18th birthday. The timing of conception in relation to a teenager's birthday determined whether she would attain her majority before her pregnancy became too advanced for her to obtain an abortion.

The decline in the abortion rate among teenagers whose pregnancy occurred $0-3$ months after their 18th birthday and the decline among those who became pregnant $0-3$ months before their birthday-and would therefore have been able to obtain an abortion by the end of their first trimester-did not differ significantly. In contrast, the decline among teenagers who conceived 3-6 months before they became 18-and would have been able to attain an abortion only in their second trimester-was $16 \%$ greater. Decreases in the birthrate did not differ significantly among the three groups.

Analyses by race and ethnic group found a significantly greater decline in the abortion rate among minors than among 18-year-olds for Hispanics and non-Hispanic whites, and a significantly greater rise in the birthrate among non-Hispanic whites. No significant differences by age were found among non-Hispanic blacks.

The decline in the odds that minors who became pregnant 3-6 months before their 18th birthday would have an abortion after enforcement of the parental notification law began was $23 \%$ greater than the decline among 18 year-olds, and the increase in their odds of having a second-trimester abortion was 34\% greater than that among 18-year-olds. No significant increase occurred among younger 17 year-olds, who would not have attained their majority until too late in pregnancy for abortion to be an option.

According to the authors, three-quarters of young women who conceive as 17 -year-olds and are exposed to a parental notification requirement give birth as 18-year-olds, and have 
thus been misclassified as adults in earlier studies. The authors avoid this source of biaswhich would lead to an underestimate of the effect of such laws-in the current study by measuring young women's age at conception rather than their age at abortion or birth

The authors acknowledge that their findings cannot be considered causal because of the observational design of the study. Nevertheless, given that the U.S. House of Representatives passed legislation in 2005 that would bar mi-

nors from consenting to an abortion in any state other than their own, and that the Senate is considered likely to approve a similar bill, the researchers argue that their results are "relevant to an assessment of the likely effect of pending legislation" extending such laws._-F. Althaus

\section{REFERENCE}

1. Joyce T, Kaestner R and Colman S, Changes in abortions and births and the Texas parental notification law, New England Journal of Medicine, 2006, 354(10): 1031-1038.

\section{Living in a Poor Neighborhood Is a Risk Factor For Overdue Pap Screening in Black Women}

Black women who live in neighborhoods with high poverty rates are more likely than their counterparts in low-poverty areas to have not been screened for cervical cancer within the past two years, according to a nationwide study. ${ }^{1}$ In addition, black women who are 40 or older or obese, who smoke or who are not college graduates have an increased likelihood of not having been screened recently. Even after adjustment for these and other individual risk factors, however, the association between neighborhood poverty and women's screening status remains.

Current clinical guidelines recommend that women younger than 65 be screened for cervical cancer every $1-3$ years. Some evidence suggests that women who live in areas with low poverty rates have higher rates of cervical cancer screening than women who live in highpoverty areas. However, it is unclear whether neighborhood-level risk factors are independent of established individual risk factors. To find out, researchers analyzed data from the Black Women's Health Study. The longitudinal study, launched in 1995, enrolled 59,090 black women aged 21-69, most of them subscribers to Essence magazine. The current analysis focused on 40,009 participants who, at baseline, were younger than 65, had not had cervical cancer or a hysterectomy, provided information on all relevant variables and had an address that could be coded by neighborhood poverty level using census data.

The outcome of interest was the self-reported length of time that had elapsed since participants' last cervical cancer screening; a screening was categorized as recent if it had occurred within two years. The researchers investigated a variety of individual-level predictors of recent screening, including age, marital status, parity, cigarette use, body mass index and family history of breast cancer. To examine neighborhood poverty, they identified the 1990 census tract (a fairly homogenous group of about 3,000 residents) that corresponded to each participant's 1995 address and determined the proportion of residents who lived in poverty in 1990. They also examined poverty rates at the state level.

Overall, $8 \%$ of participants had not received a Pap smear in the preceding two years. However, rates exceeded $10 \%$ in several subgroups of women, including those who had not attended college, never-married women, women 50 or older, and women with a body mass index of 30 or more $\mathrm{kg} / \mathrm{m}^{2}$. Professional women and those with one or two children (as opposed to nulliparous women or those with three or more children) had a reduced likelihood of not having been screened.

In a multivariate analysis, several of these individual-level factors remained significant predictors that a woman had not received a recent Pap smear-notably, education, marital status and age. Women who had not completed high school, for example, were much more likely than women who had attended graduate school to have not been screened (odds ratio, 1.8); odds ratios were also elevated, though to a lesser extent, for high school graduates (1.5) and women who had attended college but had not graduated (1.3). Women who had never married were more likely than their married counterparts to have not been screened recently (1.9), as were women who were separated (1.4), divorced (1.2) or widowed (1.4). The odds of nonscreening were substantially higher among women aged 40-49 (1.9), 50-59 (2.1) and 60-64 (2.4) than among women 29 and younger.
Cigarette use, body mass index and parity were also associated with screening status. Current smokers were more likely than nonsmokers to have not been screened recently (odds ratio, 1.2). Women who were very thin or obese were also at risk; compared with women whose body mass index was 20-24.9, those whose body mass index was less than 20 or 30 or greater had an increased likelihood of nonscreening (1.3 and 1.6, respectively). Women with one or two children were less likely than nulliparous women to be overdue for screening (0.6 and 0.8, respectively).

Further analysis revealed that neighborhood poverty rate was a significant predictor of screening status, even after the researchers controlled for individual risk factors. Women who lived in census tracts with poverty rates of at least 20\% were more likely than those who resided in tracts with poverty rates below 5\% to have not been screened (odds ratio, 1.2). Because education level was a strong predictor of screening status, the investigators examined whether neighborhood-level effects were significant among women with at least a college education; even in this group, those who lived in neighborhoods with a poverty rate greater than $20 \%$ were more likely than women who lived in census tracts with poverty rates below $5 \%$ to have not been screened (1.3). Although state of residence predicted screening status, the association did not appear to be related to state poverty levels. "It is not clear what is causing the differences between states," the researchers note

Despite the study's limitations-including a reliance on self-reports of Pap smear history and the use of a nonrandom sample of black women-the researchers observe that their results echo those of earlier studies that found that lower education, older age, obesity and smoking are associated with not having had a recent Pap test. "The study adds to the literature by demonstrating that census tract and state factors influence cervical cancer screening behaviors above and beyond individual factors," the investigators note. Given these results, they conclude that "community outreach programs should focus on high-poverty neighborhoods to decrease the proportion of black women who are not adherent to cervical cancer screening recommendations." - P. Doskoch

\section{REFERENCE}

1. Datta G et al., Individual-, neighborhood-, and state-level socioeconomic predictors of cervical carcinoma screening among U.S. black women: a multilevel analysis, Cancer, 2006, 106(3):664-669. 


\section{Many People with Herpes Use Condoms Only During Symptomatic Outbreaks}

Three-fourths of individuals who know that they have genital herpes either abstain from vaginal sex or always use condoms when they have symptoms, but only one-fifth do so when they are free of symptoms. ${ }^{1}$ In an international cross-sectional study of individuals with genital herpes who were in monogamous, heterosexual relationships, only about half knew that the infection could be transmitted between outbreaks. Participants' odds of having sex (vaginal, oral or anal) during symptomatic periods were elevated if they or their partner used an IUD, if they were Latin American or European (rather than North American) and if they had previously had an STD. Their odds also increased with the number of sex acts per month and with the number of outbreaks per year.

The study was conducted between 1998 and 2001 among clinic clients who were being screened for participation in an international trial of drug therapy to prevent transmission of genital herpes. In 17 countries in North America, Latin America and Europe, researchers gave questionnaires to individuals visiting clinics who were infected with herpes simplex virus type 2, reported having had symptoms and had partners who had never had symptomatic genital herpes. Participants provided information about their demographic characteristics and their sexual behavior (including whether they practiced abstinence and used condoms during symptomatic and asymptomatic periods), and answered questions testing their knowledge about genital herpes

Analyses were based on 1,193 individuals, $88 \%$ of whom were white and $60 \%$ of whom were female. The median age was 36 among men and 33 among women. Six in 10 participants had attended college, and seven in 10 were employed. The median number of outbreaks of genital herpes each year was five. Some $87 \%$ of participants said that they could recognize early symptoms of an outbreak.

Participants had sex with their partner a median of 6-7 times per month. During periods when they had no symptoms of genital herpes, $98 \%$ of individuals had vaginal sex, $76 \%$ had oral sex and $25 \%$ had anal sex. In contrast, during periods when they had symptoms, $40 \%$ of individuals had vaginal sex, 29\% had oral sex and $11 \%$ had anal sex
For certain types of sex, regular condom use was more common during symptomatic periods than during asymptomatic ones. Specifically, 20\% of individuals used condoms for every act of vaginal sex when they did not have symptoms, but 35\% did so when they had symptoms. Similarly, $2 \%$ always used condoms when having oral sex during asymptomatic periods, whereas $7 \%$ did so during symptomatic periods. The level of regular use of condoms when having anal sex did not vary with the presence of symptoms.

An analysis of overall changes in behavior between asymptomatic and symptomatic periods showed that most participants (89\%) engaged in some type of sex without always using condoms when they had no symptoms, and a substantial proportion of this group (38\%) continued to do so when they were symptomatic. Nonetheless, the majority of individuals modified their behavior, either abstaining from sex or always using condoms, when symptoms were present. Whereas only one-fifth (21\%) of participants abstained from or always used condoms during vaginal sex when they were free of symptoms, three-fourths (74\%) did so when they had symptoms. The pattern was similar for oral sex (26\% vs. $73 \%$ ) and anal sex ( $78 \%$ vs. $91 \%$ ).

Nearly all individuals (97\%) knew that genital herpes is sexually transmitted, and twothirds (66\%) were aware that the disease is incurable. Most knew that medication can prevent outbreaks (91\%) and can speed the healing of sores (96\%), and that condoms can help prevent the spread of the disease between partners (92\%). However, only 67\% knew that a person can still transmit the virus after a sore has completely healed, and only 53\% were aware that the virus can be transmitted between outbreaks.

In a multivariate analysis, individuals who currently used an IUD or whose partner did had higher odds of engaging in any kind of sexual activity during symptomatic periods than did nonusers (odds ratio, 3.0). The odds of having sex despite the presence of symptoms were higher among Latin American and European participants than among their North American counterparts (2.2 and 1.7, respectively), and higher among individuals who had previously had an STD than among those who had not (1.4). The odds also rose with each additional sexual act per month (1.1) and with each additional recurrence of symptoms per year (1.1).

A final, multinomial analysis examined factors associated with individuals' risk of trans- mitting genital herpes, as defined by their sexual behavior and condom use during asymptomatic and symptomatic intervals. The lowest risk (characterized by having vaginal sex only during asymptomatic periods and always using condoms) was associated with high levels of education and of knowledge about genital herpes. The highest risk (marked by frequent sexual activity with irregular condom use during both symptomatic and asymptomatic periods) was associated with being in a longterm relationship.

Study participants, the researchers acknowledge, were interested in preventing transmission of genital herpes and may not be representative of other populations. Nevertheless, although the majority of this group either abstained from sex or always used condoms when they had symptoms, their level of consistent condom use during asymptomatic periods was fairly low, suggesting that individuals with genital herpes may believe that they have little or no risk of infecting others at such times. "A focus of prevention must continue to highlight the importance of regular and consistent condom use during both symptomatic and asymptomatic periods," the researchers conclude. -S. London

\section{REFERENCE}

1. Rana RK et al., Sexual behaviour and condom use among individuals with a history of symptomatic genital herpes, Sexually Transmitted Infections, 2006, 82(1): 69-74

\section{Second-Trimester Abortion: Logistics and Lack Of Symptoms Are Factors}

By the time they suspected and confirmed their pregnancies, more than half of patients seeking a second-trimester abortion at a California clinic had already missed the opportunity to have a first-trimester abortion. ${ }^{1}$ In a study that asked women to identify factors that delayed their efforts to obtain an abortion, those who sought an abortion during the second trimester reported greater delays than first-trimester patients at each step of the process, from suspecting that they were pregnant to coming to the clinic. Moreover, logistical problems, such as difficulties in finding a provider, were widespread and contributed to delays for almost two-thirds of second-trimester patients.

Twelve percent of abortions in the United States are performed during the second 
trimester, when costs, complications and mortality are higher than in the first trimester. Because much of the literature on delays in obtaining abortions is outdated or has focused on narrow subgroups (e.g., adolescents), the researchers studied current factors associated with delay in a sample of 398 women who sought an abortion at a hospital-based clinic in San Francisco in 2001-2002; the sample was split about evenly between first- and secondtrimester patients. Before they received their abortion, participants completed a computerassisted self-interview that collected information about demographic, social and medical variables, as well as information on the timing of key steps leading up to the abortion. The primary outcome was gestational duration at the time of the procedure.

Bivariate analyses revealed numerous differences between first- and second-trimester patients. Participants who obtained secondtrimester abortions were significantly more likely than their first-trimester counterparts to have had trouble finding an abortion provider (45\% vs. $26 \%$ ), to have been referred from other clinics $(86 \%$ vs. $58 \%$ ), to have had previous second-trimester abortions (31\% vs. 15\%) and to be unsure of the date of their last menstrual period (37\% vs. $23 \%$ ). In addition, they were less likely than first-semester patients to report pregnancy symptoms, such as nausea or vomiting (68\% vs. $81 \%$ ). Roughly two-thirds of women in each group said they had been using contraceptives at the time of conception.

Mean gestational duration was 70 days greater in the second-trimester group than in the first-trimester group. About half of the difference was due to the extra time it took for second-trimester patients to suspect that they were pregnant (28 days after missing their period, compared with six days in the firsttrimester group) and to obtain a positive pregnancy test (28 days after suspecting the pregnancy, compared with 15 days in the firsttrimester group). As a result, by the time their pregnancy was confirmed, $58 \%$ of women who eventually underwent second-trimester abortions were already in their second trimester. However, durations were also longer in the second-trimester group than in the first-trimester group for each subsequent step in the process of obtaining an abortion: deciding to get an abortion, calling an abortion clinic, calling the study clinic and arriving for the abortion.

Overall, $64 \%$ of women in the first-trimester group and $86 \%$ of those in the second-trimester group cited at least one factor that delayed their abortion. Logistical problems were especially common, causing delays for $63 \%$ of secondtrimester patients and 30\% of first-trimester patients; in particular, women who had abortions in their second trimester were significantly more likely than women in their first trimester to say that they had difficulty finding a provider ( $20 \%$ vs. $7 \%$ ), were initially referred to a different clinic (47\% vs. 13\%) and had difficulty arranging transportation (10\% vs. $4 \%$ ). Nearly a third of the second-trimester group and a fifth of the first-trimester group said that a logistical factor (as opposed to emotional, financial, interpersonal or other factors) caused the most delay for them. Women in their second trimester were also significantly more likely than first-trimester patients to attribute delays to not suspecting pregnancy (34\% vs. $20 \%$ ), to problems in obtaining Medicaid coverage ( $7 \%$ vs. $2 \%$ ) and to difficulty in deciding to have the abortion (30\% vs. $20 \%$ ).

After adjustments for numerous demographic, medical, logistical, emotional and interpersonal variables, multivariate logistic regression revealed several factors associated with an increased risk of second-trimester abortion: prior second-trimester abortion (odds ratio, 5.9), difficulty in obtaining state insurance (4.4), initial referral elsewhere (4.1), difficulty in finding a provider (2.3) and uncertainty about the timing of the last menstrual period (2.3). Women had a reduced likelihood of second-trimester abortion if they had nausea or vomiting (0.4), had had a prior abortion (0.4) or used contraceptives (0.4).

The findings underscore the need for second-trimester abortion to remain legal and accessible, the researchers note, as "many women seeking second-trimester abortions simply lacked pregnancy symptoms or were unaware of their last menstrual period and therefore took a long time to recognize and test for pregnancy." Public health measures that may reduce the prevalence of second-trimester abortions include improving access to contraceptives, providing low-cost home pregnancy tests and educating patients about the importance of keeping track of menstrual periods. However, "because of the individual nature of many of the reasons for delay, it is unlikely that public health measures alone" can greatly reduce the number of late abortions. - P. Doskoch

\section{REFERENCE}

1. Drey EA et al., Risk factors associated with presenting for abortion in the second trimester, Obstetrics E Gynecology, 2006, 107(1):128-135.

\section{Bulk of Health Care Costs For People with HIV Are For Drugs, Hospitalization}

Annual expenditures on primary health care for individuals attending an outpatient HIV clinic in Alabama amount to nearly $\$ 20,000$ per patient; the drugs used for highly active antiretroviral treatment (HAART) account for more than half of the total. Costs among the sickest patients (those with the lowest CD4 cell counts) come to nearly triple those among individuals whose disease is least advanced. However, the cost of HAART is fairly consistent regardless of stage of the disease; therefore, the sicker the individual, the smaller the share of expenditures attributable to these medications. Increased expenditures for individuals with the lowest CD4 cell counts reflect mainly their need for other types of medication and for hospitalization. Provider costs represent a negligible proportion of medical care costs for HIV patients, irrespective of CD4 cell count. ${ }^{1}$

Using a database containing information on all patients receiving primary care at a university HIV clinic in the 12 months beginning March 2000, analysts examined five components of care: hospitalization (including inpatient medications, examinations and procedures), antiretroviral medications, other drugs, physician and clinic fees (for inpatient and outpatient care), and outpatient services performed at a site other than the clinic (such as home health care and laboratory studies). They determined cost data from Medicare reimbursement rates and average wholesale prices of medications.

The analyses included 635 patients who had a baseline CD4 cell count on March 1, 2000, and at least one clinic visit or hospitalization later in the study period; half were followed up for at least seven months. The majority of the cohort were male (77\%) and white (59\%); the average age was 35 years. Fifty-four percent of individuals in the cohort had private health insurance, $31 \%$ were covered by a public program and $15 \%$ lacked any health care coverage. Eight in 10 patients were receiving HAART at baseline, and data on their viral load indicate that they were generally responding well to the treatment. The analysts categorized patients by four strata of CD4 cell counts at baseline; $10 \%$ of the cohort were in the lowest stratum, and 52\% were in the highest.

Overall, annual expenditures averaged $\$ 18,640$ per patient. Of this total, $56 \%$ was at- 
tributable to antiretroviral medications, $23 \%$ to other drugs, $13 \%$ to hospital costs, $6 \%$ to outpatient services not provided at the clinic and $2 \%$ to physician or clinic costs. Expenditures totaled $\$ 13,885$ for individuals at the earliest stage of disease but rose steadily across declining strata of CD4 cell counts, reaching $\$ 36,532$ for those with the most advanced disease. Most of the disparity was accounted for by differences in costs of drugs other than antiretroviral medications ( $\$ 1,885$ for individuals with the least advanced disease, compared with $\$ 14,882$ for the sickest patients) and of hospitalization ( $\$ 1,408$ vs. $\$ 8,353$ ). By contrast, the cost of HAART was not directly related to stage of the disease and fluctuated only between about $\$ 9,407$ and $\$ 11,935$ per year; consequently, HAART represented $68 \%$ of costs for individuals with the highest CD4 cell counts, but only 30\% for those with the lowest counts. Outpatient services not delivered at the clinic accounted for 5-7\% of expenditures, depending on the stage of a patient's disease; physician and clinic costs, for $1-2 \%$.

For patients who were initially in the nextto-lowest stratum of CD4 cell counts, a decline in CD4 cell count within six months after baseline was associated with a statistically significant increase in health care expenditures; the increased expenditures were accounted for mainly by medications other than antiretroviral drugs and hospitalizations. No similar relationship was observed for the sickest patients or for those whose disease was less advanced.

The analysts comment that primary health care expenditures on individuals with HIV infection "are, paradoxically, both decreased by and driven by the use of antiretroviral medica- tions." However, the "most striking finding," in their view, was the low level of expenditures attributable to physician and clinic services. The $2 \%$ average expenditure for this component of care amounts to about $\$ 360$ per patient per year, a fraction of the $\$ 1,500-2,000$ that it costs to provide physician and clinic services. ${ }^{2}$ According to the analysts, this "meager" level of reimbursement is "inadequate to cover the cost of care provision at most HIV clinics in the United States, the majority of which are subsidized by federal and state dollars." - D. Hollander

\section{REFERENCES}

1. Chen RY et al., Distribution of health care expenditures for HIV-infected patients, Clinical Infectious Diseases, 2006, 42(2):1003-1010.

2. Infectious Diseases Society of America, Study shows AIDS drugs cost-effective, care underfunded, news release, Alexandria, VA, Feb. 27, 2006. 